\title{
Unusual ending of the second dorsal metacarpal artery as proper palmar digital arteries
}

\author{
H.F. Bianchi ${ }^{1}$, C.A. Vargas ${ }^{2}, 4$, N.E. Ottone ${ }^{3,4,5}$ \\ ${ }^{1}$ Hand Surgeon, Senior Professor, Medicine School, Buenos Aires University and IUCS Barcelo Foundation, \\ Buenos Aires, Argentina \\ ${ }^{2}$ Department of Physical Education, Education School, Universidad de La Frontera, Temuco, Chile \\ ${ }^{3}$ Laboratory of Plastination and Anatomical Techniques, Research Centre in Dental Sciences (CICO), \\ Dental School, Universidad de La Frontera, Temuco, Chile \\ ${ }^{4}$ Doctoral Programme in Morphological Sciences, School of Medicine, Universidad de La Frontera, Temuco, Chile \\ ${ }^{5}$ Centre of Excellence in Morphological and Surgical Studies (CEMyQ), Universidad de La Frontera, Temuco, Chile \\ [Received: 21 May 2017; Accepted: 18 July 2017]
}

\begin{abstract}
Background: The proper palmar digital arteries arise infrequently from dorsal metacarpal arteries. This work reports the second dorsal metacarpal artery unusual ending and a vascular variation related to the superficial palmar arch.

Materials and methods: Fresh human upper limbs routine dissection, from a male cadaver, injected with coloured elastomeric material, immersed in 10\% formalin. Results: The second dorsal metacarpal artery presented terminal branches, the index finger medial proper digital palmar artery and the middle finger lateral proper digital palmar artery. At the division point of both terminal branches, the second dorsal metacarpal artery anastomoses with a thin forth common digital palmar artery.

Conclusions: The value of this knowledge about the infrequent vascular disposition lies in vascularised flaps design using the second dorsal metacarpal artery. (Folia Morphol 2018; 77, 2: 393-396)
\end{abstract}

Key words: second dorsal metacarpal artery, proper palmar digital arteries, superficial palmar arch, vascularised flap

\section{INTRODUCTION}

The origin of the proper palmar digital arteries (PPDAs) in dorsal metacarpal arteries (DMAs) is an infrequent event. Adachi [1], Coleman and Anson [2], Earley [6] and Libersa et al. [14] mentioned this possibility, but they give only inaccurate references. The second dorsal metacarpal artery (2DMA) is important because it is the arterial vascular pedicle of the 2DMA flaps used to treat different injuries of hand and digits.

\section{MATERIALS AND METHODS}

Fresh male human cadaver, 64 years old. Right and left upper limbs were injected through brachial artery with elastomeric material using manual pressure, immersed in 10\% formalin, and dissected using standard technique and $2 \times$ magnification lenses.

\section{RESULTS}

In both hands the origin of 2DMA from the radial artery (RA) is found in the anatomical snuffbox. It passes under the extensor carpi radialis longus, surrounding the base of the second metacarpal bone, reaches the second interosseous space, between the extensors carpi radialis longus and brevis, giving muscular, osseous and cutaneous branches (Figs. 1, 2).

Following its path, it goes under the extensor indicis and the intertendinous connection between the

Address for correspondence: Dr. N.E. Ottone, Laboratory of Plastination and Anatomical Techniques, Research Centre in Dental Sciences (CICO), Dental School, Universidad de La Frontera, Temuco, Chile, e-mail: nicolas.ottone@ufrontera.cl 

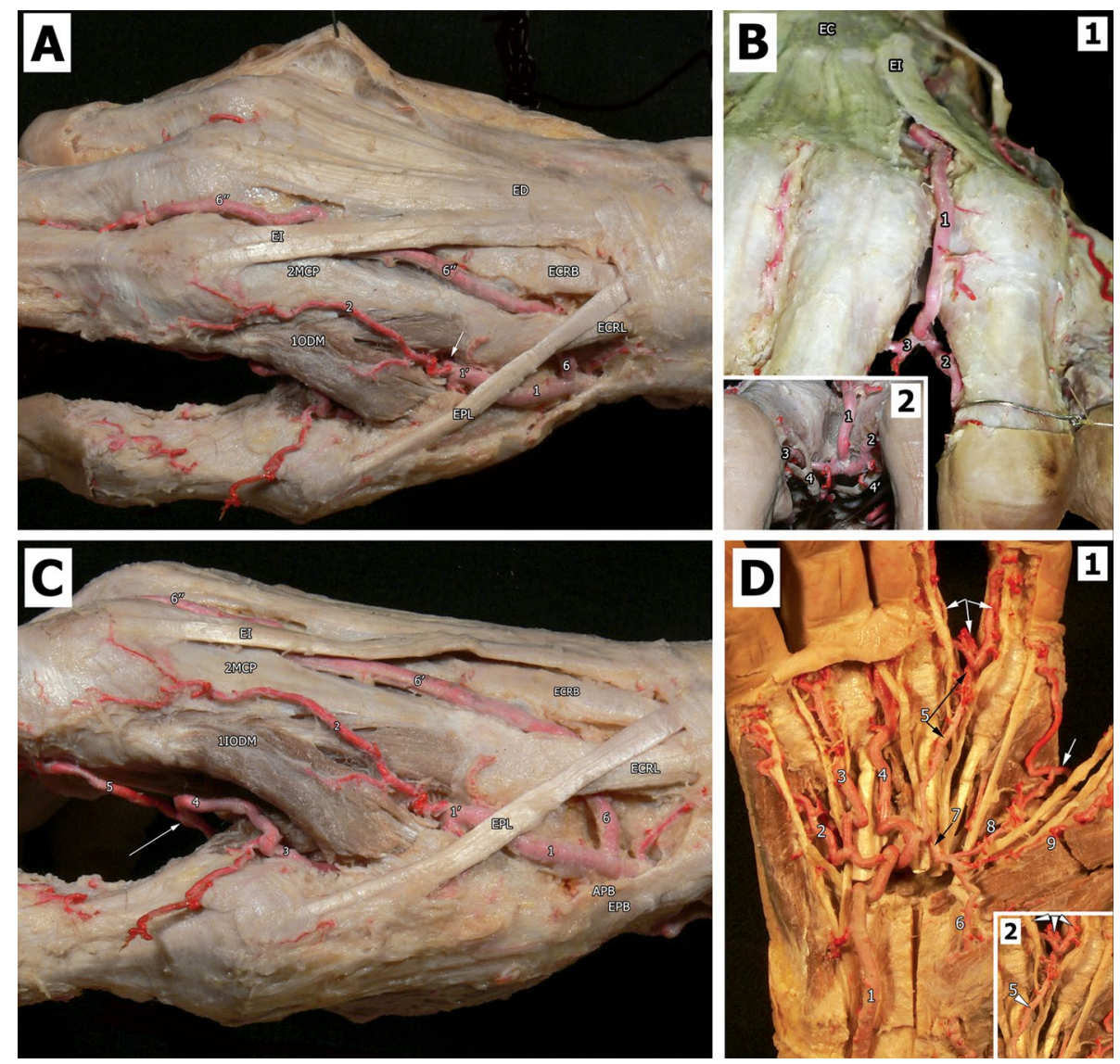

Figure 1. A. Dorsal view of the right hand. 1-1' - radial artery with 1 ' and 3 first metacarpal artery (1MCA); 2 - first dorsal metacarpal artery (1DMA); // 5 ARI; 6-6'-6" - second dorsal metacarpal artery (2DMA); APB — abductor pollicis brevis; EPB — extensor pollicis brevis; EPL - extensor pollicis longus; ECRL — extensor carpi radialis longus; ECRB - extensor carpi radialis brevis; 2MCP — second metacarpal bone; El - extensor indicis; 1IODM — first interosseous dorsal muscle; B. 1. Second web space view. 1 - 2DMA in the second web space; 2-3 - DPPA medial of index finger and lateral of middle finger; EC - extensor digitorum; El - extensor indicis. 2. Detail of the second web space. 4-4' - proper palmar digital nerves. C. Latero-dorsal view of the right hand snuffbox. 1-1' — radial artery; 2 - first dorsal metacarpal artery (1DMA); 1 and 3 - 1MCA; // 5 ARI; 6-6'-6" - second dorsal metacarpal artery (2DMA); APB — abductor pollicis brevis; EPB extensor pollicis brevis; EPL — extensor pollicis longus; ECRL — extensor carpi radialis longus; ECRB — extensor carpi radialis brevis; 2MCP — second metacarpal bone; El — extensor indicis; 1IODM — first interosseous dorsal muscle; ED — extensor digitorum. D. 1 \& 2. Palmar view of the right hand. 1 - ulnar artery; 2, 3, 4-1-2-3 common palmar digital arteries (1, 2, 3 CPDA); $5-4$ CPDA; 6 - radial palmar branch; 7 - anastomoses of radial palmar branch with 4 CPDA; 8 - anastomoses of radial palmar branch with radialis indicis artery from 1MCA (white arrow); 9 - anastomoses of radial palmar branch with proper palmar digital artery radial of the thumb; Triangular arrows: central 2DMA laterals, DPPA medial of index finger and lateral of middle finger; RIA — radialis indicis artery.

index and middle finger. In the second web space, it follows as distal perforating artery and divides in the PPDAs medial of the index finger and lateral of the middle finger. In the point of division, it anastomoses with a very thin fourth common digital palmar artery (4CDPA).

In the right hand (Fig. 1), the first, second and third common palmar digital arteries (1, 2, 3CPDAs) originate from the ulnar artery, while the 4CPDA originates from the 3CPDA. This last artery, anastomoses with the superficial palmar branch of the radial artery (SPBRA), which gives two branches: one is an anastomotic thin branch with the radial PPDA of the thumb; the other branch, anastomoses with radialis indicis artery (RIA), a branch of the first metacarpal artery (1MCA). In the left hand (Fig. 2), the ulnar artery anastomoses with the $1 \mathrm{MCA}$, at the origin point of a thin 4CPDA, similar to the right hand. The 4CPDA finishes with the 2DMA anastomosis at PPDAs division point.

\section{DISCUSSION}

The 2DMA, found in high percentages, originates from RA $[3,5,6,9,20]$, dorsal carpal arch $[1,3,7$, $9,12]$, deep palmar arch $[4,11]$, and anterior interosseous artery [7]. Following the second dorsal 


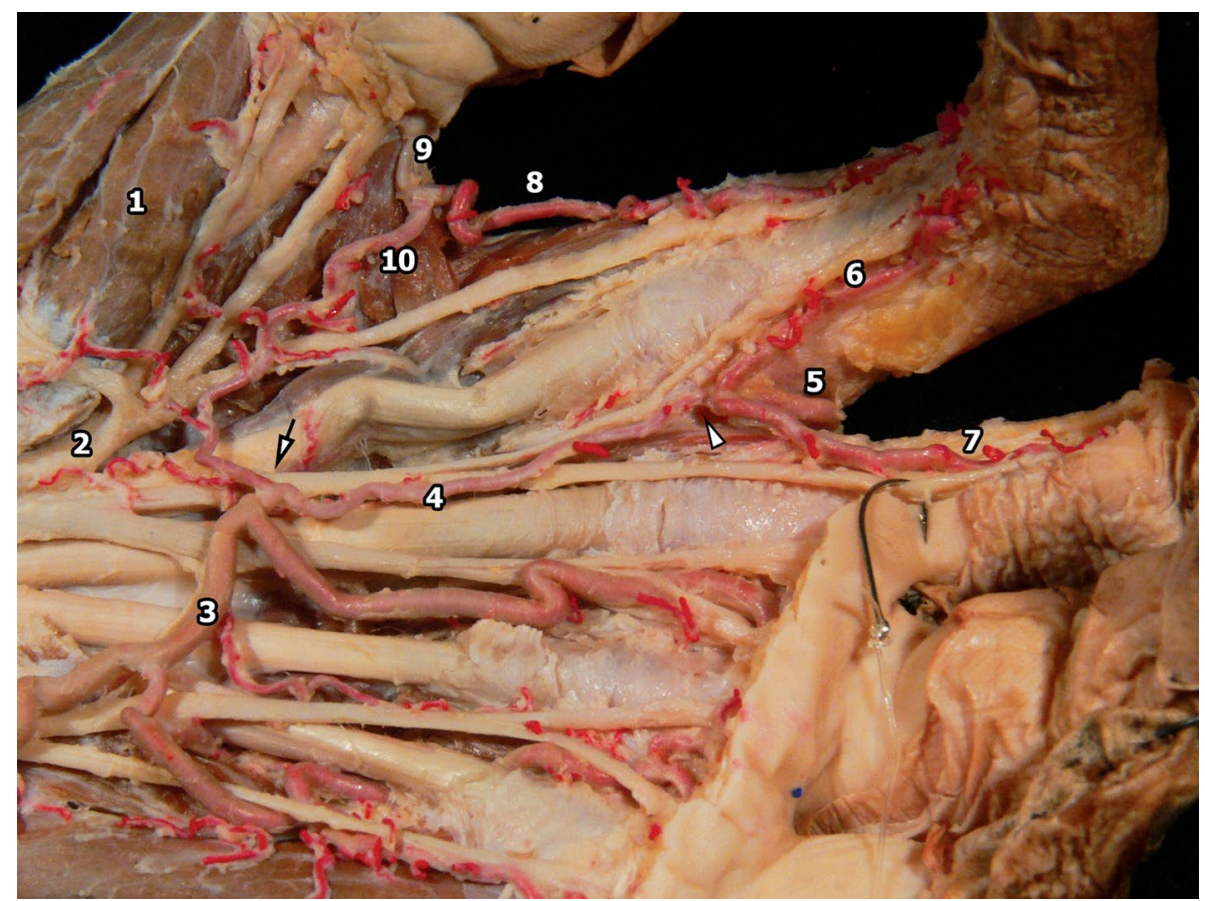

Figure 2. Palmar view of the left hand. 1 - tenar eminence; 2 - lateral branch of the median nerve with branches to thumb and index fingers; 3 - superficial palmar arch; $4-4$ CPDA (proximal arrow shows the 4CPDA anastomoses with the branch of 1MCA (10) and distal arrow shows anastomoses with 2DMA (5); 6 - medial PPDA to index finger; 7 - lateral proper palmar digital artery to middle finger; 8 radialis indicis artery; 9 - medial proper palmar digital artery of the thumb; CPDA — common palmar digital artery; DMA — dorsal metacarpal artery; MCA — metacarpal artery.

interosseous space from these different sources, gives branches to the dorsal skin of the hand, the skin of next fingers, and second metacarpal bone.

It arrives to the second web space and ends as distal perforating branch, anastomotic with branches from the deep palmar arch, which is mentioned by all the authors $[1-5,9,17]$, except Earley [6], that found it only in four specimens. Yang and Morris [19] described the perforating branches with origin in the palmar metacarpal arteries.

Adachi [1] and Libersa et al. [14] mention the 2DMA as a source of PPDA; Edwards [8] suggested that this origin could be possible due existing anastomoses at the second web space, but in his series of specimens, he did not find it. Coleman and Anson [2] described its presence limited to the first and second digital web spaces. In these papers, there are no images, drawings or schemes illustrating the descriptions.

In our study, the 4CPDA was found to be a small blood vessel in contrast with the well-developed 2DMA. Additional finding was the lack of a second palmar metacarpal artery, which compensated the low contribution of the 4CPDA.
We share the opinion of Rodríguez-Niedenführ et al. [16] regarding the development of arteries that they came from buds, initial capillary plexuses, and differentiate from proximal to distal, process that turns them into definitive blood vessels, while others disappear or undergo an incomplete development. In our case, the 4CPDA is insufficient to give the fingers blood supply and the 2PMA, that supplies the last artery, is absent (possible regression as suggested by Rodríguez-Niedenführ et al. [16]); therefore the 2DMA is increased in size to compensate the 4CPDA and becoming in the dorsal origin of the PPDA, as established by Edwards [8].

One more time, we can appreciate variations in blood vessels in the radial side of the hand as described by Edwards [8]. In the right hand, a superficial palmar arch is composed of the anastomoses between the superficial palmar branch of the radial artery (SPBRA) and the 4CPDA, which originates in the $3 C D P A$. Because of this vascular pattern, the superficial arch could be considered as complete but indirect, because the anastomoses exist, but between the SPBRA and a collateral branch of the ulnar superficial 
artery. Literature about this anatomical disposition described above was not found.

The use of the second dorsal metacarpal artery flap is a technique widely used, to cover damage areas of hand or fingers because of the high presence of the artery (93-100\%) $[6,8,9]$. We can design it as a proximal or distal vascularised cutaneous flap [3-5, $7,9,12,15,20]$ or as a vascularised bone flap $[3,13]$. Regardless of flap type, the 2DMA or the distal perforant artery must be ligated [12, 20]. In this case, this manoeuvre can be dangerous for the circulation of index and middle fingers, if 4CPDA, or another artery, is not sufficiently developed to compensate the blood supply lost. There are reports using 2DMA as a vascular pedicle, in the index pollicisation in case of congenital thumb aplasia [18].

The eco-Doppler examination is advised prior to the surgery $[4,5,9,10]$ because of the variations of the metacarpal dorsal arteries. The variation described in this case will be easy to detect, if this recommendation is followed, avoiding surprises at the moment to design the flap or planning surgery.

We were unable to find accurate descriptions of this vascular pattern in the literature, except inaccurate references without any kind of illustration. The development of vascularised flaps with the 2DMA requires the knowledge of anatomical variations of the origin and course of this artery and its connections with the superficial and deep palmar arches, no matter if they are, fortunately, of low incidence.

\section{REFERENCES}

1. Adachi B. Das Arteriensystem der Japaner. Band I. Kyoto: Maruzen Co. 1928.

2. Coleman SS, Anson BJ. Arterial patterns in the hand based upon a study of 650 specimens. Surg Gynecol Obstet. 1961; 113: 409-424, indexed in Pubmed: 13694610.

3. Dauphin N, Casoli V. The dorsal metacarpal arteries: anatomical study. Feasibility of pedicled metacarpal bone flaps. J Hand Surg Eur Vol. 2011; 36(9): 787-794, doi: 10.1177/1753193411412872, indexed in Pubmed: 21708840.

4. Dautel G, Merle M. Direct and reverse dorsal metacarpal flaps. Br J Plast Surg. 1992; 45(2): 123-130, indexed in Pubmed: 1562836.

5. de Rezende MR, Mattar Júnior R, Cho AB, et al. Anatomic study of the dorsal arterial system of the hand. Rev Hosp Clin Fac Med Sao Paulo. 2004; 59(2): 71-76, indexed in Pubmed: 15122421.
6. Earley MJ. The arterial supply of the thumb, first web and index finger and its surgical application. J Hand Surg Br. 1986; 11(2): 163-174, indexed in Pubmed: 3734551.

7. Earley MJ. The second dorsal metacarpal artery neurovascular island flap. J Hand Surg Br. 1989; 14(4): 434-440, indexed in Pubmed: 2482855.

8. Edwards EA. Organization of the small arteries of the hand and digits. Am J Surg. 1960; 99: 837-846, indexed in Pubmed: 13819401.

9. Germann G, Funk H, Bickert B. The fate of the dorsal metacarpal arterial system following thermal injury to the dorsal hand: A Doppler sonographic study. J Hand Surg Am. 2000; 25(5): 962-968, doi: 10.1053/jhsu.2000.8638, indexed in Pubmed: 11040314.

10. Healy C, Mercer NS, Earley MJ, et al. Focusable Doppler ultrasound in mapping dorsal hand flaps. Br J Plast Surg. 1990; 43(3): 296-299, indexed in Pubmed: 2190663.

11. Ikeda A, Ugawa A, Kazihara $Y$, et al. Arterial patterns in the hand based on a three-dimensional analysis of 220 cadaver hands. J Hand Surg Am. 1988; 13(4): 501-509, indexed in Pubmed: 3418051.

12. Karacalar A, Ozcan M. A new approach to the reverse dorsal metacarpal artery flap. J Hand Surg Am. 1997; 22(2): 307-310, doi: 10.1016/S0363-5023(97)80168-2, indexed in Pubmed: 9195431.

13. Khan K, Riaz M, Small JO. The use of the second dorsal metacarpal artery for vascularized bone graft. An anatomical study. J Hand Surg Br. 1998; 23(3): 308-310, indexed in Pubmed: 9665515.

14. Libersa $\mathrm{Cl}$, Francke JP, Mauppin JM, et al. The arterial supply to the palm of the hand (arteriae palmae manus). Anatomia Clinica. 1982; 4(1): 33-45, doi: 10.1007/bf01811187.

15. Quaba AA, Davison PM. The distally-based dorsal hand flap. Br J Plast Surg. 1990; 43(1): 28-39, indexed in Pubmed: 2310896.

16. Rodríguez-Niedenführ M, Burton GJ, Deu J, et al. Development of the arterial pattern in the upper limb of staged human embryos: normal development and anatomic variations. J Anat. 2001; 199(Pt 4): 407-417, indexed in Pubmed: 11693301.

17. Tasom A, Sinthubua A, Sananpanich K, et al. Cadaveric study of superficial palmar arch variations in northern Thai population by using epoxy resin injection technique. J Med Assoc Thai. 2014; 97(12): 1352-1357, indexed in Pubmed: 25764646.

18. Tonkin MA. Pollicization for congenital thumb aplasia using the second dorsal metacarpal artery as the vascular pedicle: case report. J Hand Surg Am. 2011; 36(3): 502-506, doi: 10.1016/j.jhsa.2010.11.046, indexed in Pubmed: 21295924.

19. Yang D, Morris SF. Vascular basis of dorsal digital and metacarpal skin flaps. J Hand Surg Am. 2001; 26(1): 142-146, doi: 10.1053/jhsu.2001.20967, indexed in Pubmed: 11172381.

20. Zaidenberg CR, Angrigiani C. Reverse flaps for the treatment of severe finger wounds. Rev Asoc Argent Ortop Traumatol. 1993; 58: 58-65. 